\title{
INDICE \\ ESTUDIOS INTERNACIONALES
}

Revista del Instituto de Estudios Internacionales

de la Universidad de Chile

Año XXIII

$N^{o} s 99-92$

1990

1.- INDICE CRONOLOGICO

2.- INDICE DE AUTORES

3.- INDICE DE TITULOS

4.- INDICE DE DESCRIPTORES (DE MATERIAS Y GEOGRAFICO)

Preparado por:

Biblioteca del Instituto de Estudios Internacionales 


\section{ESTUDIOS INTERNACIONALES}

\section{INDICE CRONOLOGICO}

ESTUDIOS INTERNACIONALES

AÑO XXIII 1990

\section{No89 - 1990 Enero-Marzo}

00415

Giner, Salvador; Moreno, Luis

La sociedad civil en Europa Occidental.

pp. $3-20$.

00416

Lipietz, Alain

Hacia una nueva inserción de Europa en la economía mundial.

pp. 21 - 44.

00417

Guimaraes, Roberto P.

El leviatán acorralado: continuidad y cambio en el papel del Estado en América Latina.

pp. $45-81$.

00418

Klaveren, Alberto van

Las relaciones internacionales de América Latina en la década de 1980: cambio y continuidad.

pp. $82-118$.

00419

Jaworski C., Helán

Las políticas de cooperación de Europa Occidental hacia América Latina y sus posibilidades futuras.

pp. $119-140$.

01134

Biblioteca: Instituto de Estudios Internacionales

Indice Estudios Internacionales, Año XXII, №ss. 85 - 88, 1989.

pp. $141-151$.

\section{$N^{\circ} 90$ - 1990 Abril - Junio}

00606

Varas, Augusto

Jaque a la democracia: terrorismo y anti-terrorismo en las relaciones sociales e internacionales contemporáneas.

pp. $154-165$. 
00605

Rojas Aravena, Francisco

Violencia política y orden internacional: el terrorismo en Centroamérica.

pp. $166-186$.

00601

Tokatlian, Juan Gabriel; Pardo, Rodrigo

Violencia política, paz interna y política internacional.

pp. $187-220$.

00600

García-Sayán, Diego

Terrorismo y pacificación en el Perú.

pp. $221-239$.

00602

Bustamante, Fernando

La política de Estados Unidos contra el narcotráfico y su impacto en América Latina.

pp. $240-271$.

\section{4}

Biblioteca, Instituto de Estudios Internacionales

Bibliografía: violencia política, terrorismo,' guerrilla y narcotráfico en América.

pp. $272-276$.

\section{No91 - 1990 Julio - Septiembre}

\section{5}

Tomassini, Luciano

La política internacional después del muro

pp. $281-338$.

01136

Tokatlian, Juan Gabriel; Pardo, Rodrigo

La teoría de la interdependencia: un paradigma alternativo al realișmo. pp. $339-382$.

01137

Pérez Llana, Carlos

Los noventa: una nueva agenda internacional para una nueva década. pp. $383-398$.

01138

Sunkel, Osvaldo

Perspectivas democráticas y crisis de desarrollo.

pp. $399-407$. 


\section{ESTUDIOS INTERNACIONALES}

01139

Schmied, Julie

El debate metodológico entre "clásicos" y "científicos" en las relaciones internacionales.

pp. $408-417$.

01140

Biblioteca, Instituto de Estudios Internacionales

Bibliografía: Teoría y estudio de las relaciones internacionales.

pp. $418-421$.

\section{Y०92 - 1990 Octubre - Diciembre}

01126

Mols, Manfred

Comunidad Europea-Asean: ¿un modelo de cooperación interregional? pp. $424-443$.

01127

García Menéndez, José Rạmón

Estilos de crecimiento económico periférico e inserción de América Latina en el mercado mundial. pp. $444-463$.

01128

Moneta, Carlos J.

La teoriá de las relaciones internacionales: el realismino y sự límites: pp. $464-498$.

01129

Pinochet de la Barra, Oscar

Antártica, un coṇtinente para el tercer milenio.

pp. $499-511$.

01130

Hofmeister, Wilhelm

La resolución de la cuestión alemana: su dimensión nacional e internàciotial.

pp. $512-531$, incluye anexo.

01131

Fermandois, Joaquíi.

Expansion of third world navies; de: Michacl A. Morris (Reseña).

pp. $532-537$.

01132

Tomassini, Luciano

International Relations Theory: trealism, plụralism, globalism, de: Paul R. Viotti y Mark Y. Kauppi (Reseña).

pp. $538-539$. 
Biblioteca I. E. I. / Indice: Estudios Internacionales...

01133

Biblioteca, Instituto de Estudios Internacionales

Bibliografía: Alemania 1988-1990.

pp. $540-543$.

\section{INDICE DE AUTORES \\ ESTUDIOS INTERNACIONALES \\ AÑOXXIII 1990}

Biblioteca. Instituto de Estudios Internacionales.

1133 Biblioteca. Instituto de Estudios Internacionales.

Bibliografía: Alemania 1988-1990.

No92, Octubre - Diciembre, pp. 540 - 543.

BIBLIOGRAFIA; ALEMANIA; REUNIFICACION; EUROPA

OCCIDENTAL.

1140 Biblioteca. Instituto de Estudios Internacionales.

Bibliografa: Teoría y estudio de las relaciones internacionales.

No91, Julio - Septiembre, pp. 418 - 421.

BIBLIOGRAFIA; RELACIONES INTERNACIONALES-ESTUDIO Y

ENSEÑANZA; RELACIONES INTERNACIONALES-TEORIA.

00604 Biblioteca. Instituto de Estudios Internacionales.

Bibliografía: violencia política, terrorismo, guerrilla y narcotráfico en América.

NN990, Abril -Junio, pp. 272 - 276.

BIBLIOGRAFIA; TERRORISMO; GUERRILLA; VIOLENCIA; DROGAS; AMERTCA.

1134 Biblioteca. Instituto de Estudios Internacionales.

Indice Estudios Internacionales, Año XXII, Nºs 85-88, 1989.

№89, Enero-marzo, pp. 141 - 151.

INDICE.

\section{Bustamante, Fernando}

0602 Bustamante, Fernando

La política de Estados Unidos contra el narcotráfico y su impacto en América Latina. 
NN90, Abril - Junio, pp. 240 - 271.

ESTADOS UNIDOS; AMERTCA DEL NORTE; AMERICA LATINA;

DROGAS; POLITTCA.

\section{Fermandois, Joaquín}

1131 Fermandois, Joaquín

Expansion of third world navies, de: Michael A. Morris (Reseña) No92, Octubre - Diciembre, pp. 532 - 537. //Reseña del libro: "Expansion of third world navies", Morris, Michael A.// RESEÑA DE LIBROS.

\section{García Menéndez, José Ramón}

1127 García Menéndez, José Ramón

Estilos de crecimiento económico periférico e inserción de América Latina en el mercado mundial. No92, Octubre - Diciembre, pp. 444 - 463. AMERICA LATINA; CRECIMIENTO ECONOMICO; COMERCIO INTERNACIONAL; RELACIONES ECONOMICAS.

\section{García-Sayán, Diego}

0600 García-Sayán, Diego

Terrorismo y pacificación en el Perú

№90, Abril - Junio, pp. 221 - 239.

TERRORISMO; AMERICA LATINA; PERU; DEMOCRACIA.

\section{Giner, Salvador}

0415 Giner, Salvador; Moreno, Luis

La sociedad civil en Europa Occidental

No89, Enero - Marzo, pp. 3- 20.

EUROPA OCCIDENTAL; CLASES SOCIALES; SOCIEDAD.

\section{Guimaraes, Roberto $P$}

0417 Guimaraes, Roberto P.

El leviatán acorralado: continuidad y cambio en el papel del Estado en América Latina.

№89, Enero - Marzo, pp. 45 - 81.

AMERICA LATINA; DESARROLLO ECONOMICO;

DEMOCRATTZACION; DESARROLLO SOCIAL.

\section{Hofmeister, Wilhelm}

1130 Hofmeister, Wilhelm

La resolución de la cuestión alemana: su dimensión nacional e internacional. 
Biblioteca I. E. I. / Indice: Estudios Internacionales...

NN92, Octubre - Diciembre, pp. 512 - 531 (incluye anexo). //Anexo: Cronología de los acontecimientos en el camino hacia la unidad alemana, pp. 525-531//.

EUROPA OCCIDENTAL; ALEMANIA; REUNIFICACION; RELACIONES INTERNACIONALES.

\section{Jaworski C., Helán}

0419 Jaworski C., Helán

Las políticas de cooperación de Europa Occidental hacia América Latina y sus posibilidades futuras.

№89, Enero - Marzo, pp. 119 - 140.

AMERICA LATINA; EUROPA OCCIDENTAL; COOPERACION ECONOMICA; COOPERACION TECNOLOGICA.

\section{Klaveren, Alberto van}

0418 Klaveren, Alberto van

Las relaciones internacionales de América Latina en la década de 1980: cambio y continuidad.

№89, Enero - Marzo, pp. 82 - 118.

AMERICA LATINA; RELACIONES INTERNACIONALES; ESTADOS UNIDOS; EUROPA OCCIDENTAL; ASIA; JAPON; AMERICA DEL NORTE; TERCER MUNDO; COOPERACION REGIONAL.

\section{Lipietz, Alain}

0416 Lipietz, Alain

Hacia una nueva inserción de Europa en la economía mundial. №89, Enero - Marzo, pp. 21 - 44.

EUROPA; ECONOMIA INTERNACIONAL; COMERCIO INTERNACIONAL; COMUNDDAD ECONOMICA EUROPEA.

\section{Mols, Manfred}

1126 Mols, Manfred

Comunidad Europea-Asean: ¿un modelo de cooperación interregional?

No92, Octubre - Diciembre, pp. 424 - 443.

ASEAN; ASIA; COMUNIDAD ECONOMICA EUROPEA; EUROPA OCCIDENTAL; COOPERACION ECONOMICA; RELACIONES ECONOMICAS.

\section{Moneta, Carlos J.}

1128 Moneta, Carlos.J.

La teoría de las relaciones internacionales: el realismo y sus límites. 


\section{ESTUDIOS INTERNACIONALES}

N92, Octubre - Diciembre, pp. 464 - 498.

RELACIONES INTERNACIONALES-TEORIA.

\section{Moreno, Luis}

0415 Giner, Salvador; Moreno, Luis

La sociedad civil en Europa Occidental.

N89, Enero - Marzo, pp. 3 - 20.

EUROPA OCCIDENTAL; CLASES SOCIALES; SOCIEDAD.

\section{Pardo, Rodrigo}

1136 Tokatlian, Juan Gabriel; Pardo, Rodrigo

La Teoría de la interdependencia: un paradigma alternativo al realismo.

No91, Julio - Septiembre, pp. 339 - 382.

RELACIONES INTERNACIONALES-TEORIA.

0601 Tokatlian, Juan Gabriel; Pardo, Rodrigo

Violencia política, paz interna y política internacional.

No90, Abril - Junio, pp. 187 - 220.

VIOLENCIA; COLOMBIA; AMERICA LATINA; POLITICA Y GOBIERNO.

\section{Pérez Llana, Carlos}

\section{Pérez Llana, Carlos}

Los noventa: una nueva agenda internacional para una nueva década.

No91, Julio - Septiembre, pp. 383 - 398.

ORDEN MUNDIAL; RELACIONES INTERNACIONALES; POLITICA MUNDIAL; RELACIONES INTERNACIONALES-TEORIA.

\section{Pinochet de la Barra, Oscar}

1129 Pinochet de la Barra, Oscar

Antártica, un continente para el tercer milenio.

№92, Octubre - Diciembre, pp. 499 - 511.//Conferencia dada por el autor en Roma, Pontificia Universidad Salesiana, 1989, 19 de Octubre//.

ANTARTICA; RECURSOS NATURALES; PROTECCION AMBIENTAL; FUTURO.

\section{Rojas Aravena, Francisco}

0605 Rojas Aravena, Francisco

Violencia política y orden internacional: el terrorismo en Centroamérica. 
Biblioteca I. E. I. / Indice: Estudios Internacionales...

Neg0, Abril - Junio, pp. 166 - 186.

VIOLENCIA; TERRORISMO; AMERICA CENTRAL Y CARIBE; ORDEN MUNDIAL; ESQUIPULAS II.

\section{Schmied, Julie}

1139 Schmied, Julie

El debate metodológico entre "clásicos" y "científicos" en las relaciones internacionales.

No91, Julio - Septiembre, pp. 408 - 417.

RELACIONES INTERNACIONALES-TEORIA; METODOLOGIA.

\section{Sunkel, Osvaldo}

1138 Sunkel, Osvaldo

Perspectivas democráticas y crisis de desarrollo.

No91, Julio - Septiembre, pp. 399 - 407.

AMERICA LATINA; DEMOCRATTZACION; DESARROLLO SOCIAL; DESARROLLO ECONOMICO.

\section{Tokatlian, Juan Gabriel}

1136 Tokatlian, Juan Gabriel; Pardo, Rodrigo

La teoría de la interdependencia: un paradigma alternativo al realismo.

№91, Julio - Septiembre, pp. 339 - 382.

RELACIONES INTERNACIONALES-TEORIA.

0601 Tokatlian, Juan Gabriel; Pardo, Rodrigo

Violencia política, paz interna y política internacional.

№90, Abril - Junio, pp. 187 - 220.

VIOLENCIA; COLOMBIA; AMERICA LATINA; POLITICA Y

GOBIERNO.

\section{Tomassini, Luciano}

1132 Tomassini, Luciano

International Relations Theory: realism, pluralism, globalism de:

Paul R. Viotti y Mark V. Kauppi (Reseña).

№92, Octubre - Diciembre, pp. 538 - 539. //Reseña del libro:

"International Relations Theory: realism, pluralism, globalism, Viotti, Paul R. y Mark V. Kauppi//.

RESENA DE LIBROS.

1135 Tomassini, Luciano

La política internacional después del muro.

№91, Julio - Septiembre, pp. 281 - 338.

RELACIONES INTERNACIONALES-TEORIA; POLITICA INTERNA- 
CIONAL; POLITICA MUNDIAL; RELACIONES INTERNACIONALES; ORDEN MUNDIAL.

\section{Varas, Augusto}

0606 Varas, Augusto

Jaque a la democracia: terrorismo y anti-terrorismo en las relaciones sociales e internacionales contemporáneas.

№90, Abril - Junio, pp. 154 - 165.

TERRORISMO; RELACIONES INTERNACIONALES; DEMOCRACIA.

\section{INDICE DE TITULOS}

ESTUDIOS INTERNACIONALES

AÑOXXIII 1990

\section{Antártica, un continente para el tercer milenio.}

1129 Pinochet de la Barra, Oscar

Antártica, un continente para el tercer milenio.

No92, Octubre - Diciembre, pp. 499 - 511.

//Conferencia dada por el autor en Roma, Pontificia Universidad Salesiana, 1989, 19 de Octubre//.

\section{Bibliografía: Alemania 1988-1990}

1133 Biblioteca. Instituto de Estudios Internacionales.

Bibliografía: Alemania 1988-1990.

№92, Octubre - Diciembre, pp. 540 - 543.

Bibliografia: Teoría y estudio de las relaciones internacionales.

1140 Biblioteca. Instituto de Estudios Internacionales.

Bibliografía: Teoría y estudio de las relaciones internacionales.

Ne91, Julio - Septiembre, pp. 418 - 421.

Bibliografia: violencia política, terrorismo, guerrilla y narcotráfico en América.

0604 Biblioteca. Instituto de Estudios Internacionales.

Bibliografía: violencia política, terrorismo, guerrilla y narcotráfico en América.

No90, Abril - Junio, pp. $272-276$. 
Comunidad Europea-Asean: un modelo de cooperación interregional?

\section{Mols, Manfred}

Comunidad Europea-Asean: ¿un modelo de cooperación interregional? N 92 , Octubre - Diciembre, pp. 424 - 443.

El debate metodológico entre "clásicos" y "científicos" en las relaciones internacionales.

1139 Schmied, Julie

El debate metodológico entre "clásicos" y "científicos" en las relaciones internacionales.

No91, Julio-septiembre, pp. 408 - 417.

Estilos de crecimiento económico periférico e inserción de América Latina en el mercado mundial.

1127 García Menéndez, José Ramón

Estilos de crecimiento económico periférico e inserción de América Latina en el mercado mundial.

№92, Octubre-diciembre, pp. 444 - 463.

Expansion of third world navies de: Michael A. Morris (Reseña)

1131 Fermandois, Joaquín

Expansion of third world navies de: Michael A. Morris (Reseña).

No92, Octubre - Diciembre, pp. 532 - 537.

//Reseña del libro: "Expansion of third world navies", Morris, Michael A.//

Hacia una nueva inserción de Europa en la economía mundial 0416 Lipietz, Alain.

Hacia una nueva inserción de Europa en la economía mundial. No89, Enero - Marzo, pp. 21 - 44.

Indice Estudios Internacionales, Año XXI, Ns. 85-88, 1989

1134 Biblioteca. Instituto de Estudios Internacionales.

Indice Estudios Internacionales, Año XXII, Ns. 85-88, 1989.

№89, Enero - Marzo, pp. 141 - 151.

International Relations Theory: realism, pluralism, globalism de: Paul R. Viotti y Mark V. Kauppi (Reseña).

1132 Tomassini, Luciano

International Relations Theory: realism, pluralism, globalism de: Paul R.

Viotti y Mark V. Kauppi (Reseña).

No92, Octubre - Diciembre, pp. 538 - 539. 
ESTUDIOS INTERNACIONALES

//Reseña del libro: "International Relations Theory: realism, pluralism, globalism, Viotti, Paul R. y Mark V. Kauppi//

Jaque a la democracia: terrorismo y anti-terrorismo en las relaciones sociales e internacionales contemporáneas

0606 Varas, Augusto

Jaque a la democracia: terrorismo y anti-terrorismo en las relaciones sociales e internacionales contemporáneas.

NN90, Abril - Junio, pp. 154 - 165.

El leviatán acorralado: continuidad y cambio en el papel del Estado en América Latina

0417 Guimaraes, Roberto P.

El leviatán acorralado: continuidad y cambio en el papel del Estado en América Latina.

№89, Enero - Marzo, pp. 45 - 81.

Los noventa: una nueva agenda internacional para una nueva década

1137 Pérez Llana, Carlos

Los noventa: una nueva agenda internacional para una nueva década.

№91, Julio - Septiembre, pp. 383 - 398.

Perspectivas democráticas y crisis de desarrollo

1138 Sunkel, Osvaldo.

Perspectivas democráticas y crisis de desarrollo.

№91, Julio - Septiembre, pp. 399 - 407.

La política de Estados Unidos contra el narcotráfico y su impacto en América Latina

0602 Bustamante, Fernando

La política de Estados Unidos contra el narcotráfico y su impacto en América Latina.

No90, Abril - Junio, pp. 240 - 271.

\section{La política internacional después del muro}

1135 Tomassini, Luciano.

La política internacional después del muro.

No91, Julio - Septiembre, pp. 281 - 338. 
Biblioteca I. E. I. / Indice: Estudios Internacionales...

Las políticas de cooperación de Europa Occidental hacia América Latina y sus posibilidades futuras

0419 Jaworski C., Helán

Las políticas de cooperación de Europa Occidental hacia América Latina y sus posibilidades futuras.

№89, Enero - Marzo pp. 119 - 140.

Las relaciones internacionales de América Latina en la década de 1980: cambio y continuidad

0418 Klaveren, Alberto van

Las relaciones internacionales de América Latina en la década de 1980: cambio y continuidad.

№89, Enero - Marzo, pp. 82 - 118.

La resolución de la cuestión alemana: su dimensión nacional e internacional

1130 Hofmeister, Wilhelm

La resolución de la cuestión alemana: su dimensión nacional e internacional.

№92, Octubre - Diciembre, pp. 512 - 531 (incluye anexo).

//Anexo: Cronología de los acontecimientos en el camino hacia la unidad alemana, pp. 525-531//

\section{La sociedad civil en Europa Occidental}

0415 Giner, Salvador; Moreno, Luis

La sociedad civil en Europa Occidental. №89, Enero - Marzo, pp. 3 - 20.

La teoría de la interdependencia: un paradigma alternativo al realismo

1136 Tokatlian, Juan Gabriel; Pardo, Rodrigo

La teoría de la interdependencia: un paradigma alternativo al realismo. №91, Julio - Septiembre, pp. 339 - 382.

La teoría de las relaciones internacionales: el realismo y sus límites 1128 Moneta, Carlos J.

La teoría de las relaciones internacionales: el realismo y sus límites. No92, Octubre - Diciembre, pp. 464 - 498.

\section{Terrorismo y pacificación en el Perú}

0600 García-Sayán; Diego

Terrorismo y pacificación en el Perú. №90, Abril - Junio, pp. 221 - 239. 
Violencia política y orden internacional: el terrorismo en Centroamérica.

0605 Rojas Aravena, Francisco

Violencia política y orden internacional: el terrorismo en Centroamérica.

Ne90, Abril - Junio, pp. 166 - 186.

Violencia política, paz interna y política internacional

0601 Tokatlian, Juan Gabriel; Pardo, Rodrigo

Violencia política, paz interna y política internacional.

Ne90, Abril - Junio, pp. 187 - 220.

\section{INDICE DE DESCRIPTORES}

ESTUDIOS INTERNACIONALES

AÑ $O X X I I I 1990$

ALEMANIA:

1130,1133

AMERICA:

0604

AMERICA CENTRAL Y CARIBE:

0605

AMERICA DEL NORTE:

0418, 0602

AMERICA LATINA:

0417, 0418, 0419, 0600, 0601,

$0602,1127,1138$

ANTARTICA:

1129

ASEAN:

1126

ASIA;

0418,1126

BIBLIOGRAFIA:

0604, 1133, 1140

CLASES SOCIALES:

0415
COLOMBIA:

0601

COMERCIO INTERNACIONAL:

0416, 1127

COMUNIDAD ECONOMICA EURO-

PEA:

0416,1126

COOPERACION ECONOMICA:

0419,1126

COOPERACION REGIONAL:

0418

COOPERACION TECNOLOGICA

0419

CRECIMIENTO ECONOMIĊO:

1127

DEMOCRACIA:

0600,0606

DEMOCRATIZACION:

0417,1138

DESARROLLO ECONOMICO:

0417, 1138 
Biblioteca I. E. I. / Indice: Estudios Internacionales...

DESARROLLO SOCLAL:

0417, 1138

DROGAS:

0602,0604

ECONOMIA INTERNACIONAL:

0416

ESQUIPULAS II:

0605

ESTADOS:

0417

ESTADOS UNIDOS:

0418, 0602

EUROPA:

0416

EUROPA OCCIDENTAL:

0415, 0418, 000419, 001126, 001130,001133

FUTURO:

1129

GUERRILLA:

0604

INDICE:

1134

JAPON:

0418

METODOLOGIA:

1139

ORDEN MUNDIAL:

$0605,1135,1137$

PERU:

0600

POLITICA:

0602
POLTTICA INTERNACIONAL: 1135

POLITTCA MUNDIAL:

1135,1137

POLITICA Y GOBIERNO:

0601

PROTECCION AMBIENTAL:

1129

RECURSOS NATURALES:

1129

RELACIONES ECONOMICAS:

1126,1127

RELACIONES INTERNACIONALES:

0418, 0606, 1130, 1135, 1137

RELACIONES INTERNACIONALESESTUDIO Y ENSENAANZA:

1140

RELACIONES INTERNACIONALESTEORIA:

$1128,1135,1136,1137,1139$, 1140

RESEÑA DE LIBROS:

1131,1132

REUNIFICACION:

1130,1133

SOCIEDAD:

0415

TERCER MUNDO:

0418

TERRORISMO:

$0600,0604,0605,0606$

VIOLENCIA:

0601, 0604, 0605 